\title{
Control and Quality of Data: Case Study in a USA Aviation Company
}

\author{
KARLA ESTEFANÍA ZUBIATE-PÉREZ (B.S.) \\ Universidad Autónoma de Chihuahua, México. \\ Email: karlazubiatep@gmail.com \\ Tel: +13166891716 \\ ANA ISABEL ORDOÑEZ-PARADA (PH.D) \\ Universidad Autónoma de Chihuahua, México. \\ Email: aordonez@uach.mx \\ Tel: +5216142393213
}

\begin{abstract}
All manufacturing processes involve the flow of two important components: materials and information. The production scheduling, parts traceability and quality non-conformities are kept and permanently recorded at the Phosphoric Acid Anodizing area in one of the plants of a small and medium-sized aircrafts' company in the US. The information flow within this area is a key point in the sustainability and quality of the manufacturing process. The objective of the present study was to analyze the relationship between the quantity of produced orders and the fidelity and quality of the manufacturing records among the different Production control systems used in the manufacturing plant. The study was mixed, applied, nonexperimental, correlational and cross-sectional. The independent variable was the quantity of production orders released to the Phosphoric Acid Anodizing area, while the dependent variable was the quantity of quality non-conformances found in the different Production control systems. By analyzing a probabilistic sample of 3,079 production orders, it was proven that the records kept in the different control systems are not related between them. Nonetheless, it was proven that there is a correlation between the fluctuation of the quantity of released production orders and the quantity of quality non-conformances recorded in the control systems.
\end{abstract}

Keywords: Manufacturing, Quality Non-conformances, Production Control, Control System, Phosphoric Acid Anodizing, Aircraft Company.

\section{Introduction}

The creation of more efficient and effective manufacturing processes is driven by the existing competition between companies around the globe in the search of better products and services. In the aerospace industry schedule, cost and quality are key factors in the design, manufacture and delivery of products in accordance to the market needs. The Phosphoric Acid Anodizing area, located in a small and medium-sized aircrafts' aviation company in the United States of America, works under specific production processes guidelines. This production line has the purpose of anodizing or preparing the surfaces of sheet metal and other metallic components to complete a metallic bond further down the production line.

The Phosphoric Acid Anodizing area was established in the 1990s decade and worked only with written documentation and the rudimentary use of computers to keep track of the production. In early 2000's, the company decided to incur in the use of Systems Applications and Products (SAP) software as a data 
processor for different departments. Even though this change affected the Phosphoric Acid Anodizing area, SAP did not meet all production requirements; therefore, handwritten formats remained active. In mid2010's there was an increase of the quantity of Quality non-conformances or defects, meaning the parts had to be reworked, hence redistributed through the production line.

In order to keep traceability of these parts a new software, called FlexTime, was developed, which had the purpose of automating the process and keep track of Production orders and individual parts running through the production line. Nonetheless, FlexTime did not work as expected and some of the traceability had still to be done through handwritten formats and SAP software. Nowadays, these three independent Production control systems are still been used, which causes poor traceability and misconnected and unreliable data.

The general objective of this research was to analyze the relation between the quantities of production orders released to the Phosphoric Acid Anodizing production line and the quality of the records kept through the different Production control systems used in such area: Systems Applications and Products, handwritten formats and FlexTime. The specific objectives were to determine the quantity of Quality nonconformances found when the quantity of released production orders increased and to define if the electronic Production control systems contain more accurate information, compared to the handwritten records. Related literature and studies were reviewed in order to sustain the conduced study; the methodologic design of the study was mixed, applied, non-experimental, cross-sectional and correlational.

\section{Anodizing}

The anodizing practice refers to the electrolytic process where the natural oxide layer increases its thickness significantly (Pennisi, 1999), which prevents metal corrosion and abrasion caused by atmospheric agents (Giudice \& Pereyra, 2009, pág. 3). The anodizing process in the subject company takes place to prepare the surfaces to be bonded to other metal parts further down the production line. Morris (2011) defines the chemical bond characteristics of metal as Metal Bond, where two metals adhere to each other by sharing valence electrons. Such process is promoted by preparing the involved components and applying heat and pressure.

\section{Requirements in the arrangement of the parts to be anodized}

The nature of the anodizing process establishes that the aluminum parts must go through a series of chemicals treatments which promote the increase of the thickness of the oxide layer on the surface of the part. The technique used to get the parts submerged is by attaching them, via metal wires, to racks that will allow the parts to be submerged in the chemicals. García Pérez (2006) mentions that the anodizing, since it is an electrochemical process, requires special attention in the way electric conductivity flows towards the parts to be anodize. Consequently, the parts must be disposed in a specific configuration to assure the parts will be easily detached from the rack once the anodizing is done, but still be able to have the current flowing through them while they are still in the tank.

\section{Manufacturing Defects}

The quality non-conformances are defined as manufacturing defects. The acceptance criteria for anodized parts should have the following characteristics: The anodizing layer must be uniform, the aluminum parts must show an even color change, the components should be free of burns, damage and must not show reddish or brown particles (Brown, 2016). Among the different defects found in anodized parts Hufnagel (1992) lists incorrect use of tools, defective finish in metallic surfaces, insufficient degreaser and burned greases. 


\section{Production Control}

Manufacturing processes involve the flow of two major components: materials and information. In the case of the Phosphoric Acid Anodizing process, the information flow constitutes a key element to the success of the manufacturing process (Sipper \& Bulfin, 1998). In any inline production system the product moves from one station to the next in a standardized itinerary and flow (Prado, 1992). The anodizing process requires the parts to be immerse a specific time in each one of the chemicals contained in the tanks (Brown, 2016); therefore any anodizing production control system should be designed based in time slots. This way, the action of the chemicals over the parts would be continuous and controlled.

\section{Traceability}

The traceability through the manufacturing process relies on the clients' needs, the industry standards or on defined legislations (Monette \& Van Bogart, 2011). An example of the importance of the traceability was observed in 2006 (Eisler, 2017), when 9.6 million of laptops ignited due to the use of defective Sony batteries (Monette \& Van Bogart, 2011). In the aerospace industry, companies should all have something in common: the commitment to the quality and conformity of their products (Di Natale, 2016).

\section{Monitoring}

The work in process (WIP) is present in every manufacturing process where the raw materials are transformed into finished products (Cuatrecasas-Arbós, Fortuny-Santos, Ruiz-de-Arbulo-López, \& VintróSanchez, 2015). In the Phosphoric Acid Anodizing area, the WIP is defined as all the components going through the tanks in order to be chemically treated. Systems Applications and Products (SAP) system permits a continuous monitoring of each of the work orders going through each of the production stations.

\section{Production Control Systems}

Systems Applications and Products (SAP) system is the software used as data processor for different departments such as Finance, Quality, Engineering, Purchasing and Production, and keeps precise and lasting information records (Soto, 2017). Inventories, production planning and logistics are managed through SAP system, which is an enterprise resource planning tool that translates the clients' requirements into specific production plans (Jiménez Luengo, 2010). FlexTime was designed to automatize the Phosphoric Acid Anodizing production line based in time and taking production orders as the input to trace on which part of the process they are at. Handwritten formats are used to record discrepancies and deviations that neither SAP nor FlexTime can track.

The research hypotheses were the following:

\section{General Hypothesis}

1. The increase of released production orders increases the discrepancy between the quality nonconformances records kept in the different Production control systems used in the Phosphoric Acid Anodizing area.

\section{Specific Hypotheses}

1. The quantity of quality non-conformances found in the anodized components is directly proportional to the quantity of released Production orders.

2. The electronic Production control systems keep more quality non-conformances records than the handwritten formats. 


\section{Materials and Methods}

The study was mix, non-experimental, correlational and cross-sectional.

\section{Independent variable}

Production orders released to the Phosphoric Acid Anodizing area

\section{Dependent variable}

Quantity of Quality non-conformances found in the Production orders recorded in the different Production control systems

The population was composed by 14,777 production orders released to the Phosphoric Acid Anodizing between April and September 2017. The sampling frame was built by using the data available in the Systems Applications and Products (SAP) system. The analysis units were all the production orders released and processed through the Phosphoric Acid Anodizing area, exclusively for aircrafts approved by the Federal Aviation Administration (FAA). Based on these criteria, there were 14,321 production orders used to select the population sample. The size of the sample was defined under the arrangement of the variation of released production orders per month; therefore there were six samples.

Table 1. Definition of monthly samples sizes

\begin{tabular}{|c|c|c|c|}
\hline Month & $\begin{array}{c}\text { Total quantity of released } \\
\text { production orders (N) }\end{array}$ & $\mathbf{n}$ & \multicolumn{2}{|c|}{$\mathbf{n}^{\prime}$} \\
\hline April & 2,691 & & 527 \\
\hline May & 2,522 & & 520 \\
\hline June & 2,458 & \multirow{3}{*}{655} & 517 \\
\hline July & 1,892 & & 487 \\
\hline August & 2,310 & & 511 \\
\hline September & 2,448 & 517 \\
\hline
\end{tabular}

Source: Systems Applications and Products (SAP) system records and proposed calculations

The Table 1 shows the variation of released production orders to the area per month. By using the resulting data from the Finite and Infinite populations' equations, a statistical confidence level of $99 \%$, an error margin of $5 \%$ and a success probability of 0.5 , a total of 3,079 production orders was defined as the sample size.

\section{Results}

The independent variable, as previously mentioned, was the Production orders released to the Phosphoric Acid Anodizing area. Such variable fluctuates with the market's demand which cannot be controlled. This variable was identified through the analysis of the released production orders generated in the Systems Applications and Products (SAP) system during the months of April through September of 2017.

The dependent variable was the quantity of Quality non-conformances found in the Production orders recorded in the different Production control systems. This variable was identified when the following Quality non-conformances were found: dents, parts too close to one another, contamination, corrosion, finger prints on the parts surfaces, incorrect parts grip, acid stains, water stains, black stains, reddish stains, wire marks, holes, burns, scratches, adhesive tape residues and markers remains. 
Table 2. Production Control systems characteristics

\begin{tabular}{|c|c|c|c|}
\hline Traceability & SAP & FlexTime & $\begin{array}{c}\text { Handwritten } \\
\text { formats }\end{array}$ \\
\hline Parts & No & No & Yes \\
\hline Reworks & Yes & No & Yes \\
\hline Racks & No & Yes & Yes \\
\hline Times & Yes & Yes & No \\
\hline \multicolumn{4}{|c}{ Source: Prepared by the author } \\
\end{tabular}

Table 2 shows the traceability capabilities the different Control systems are able to record. All these aspects need to be logged for each Production order that goes through the production line but there is a notorious deviation on the information logging; therefore the users need to recur to these three different control systems to make sure all the required information has been recorded. This process deviation requires effective labor time and represents lack of synchronization in the data gathering process.

Table 3. Monthly Production orders distributions

\begin{tabular}{|c|c|}
\hline Month & Quantity of orders \\
\hline April & 527 \\
\hline May & 520 \\
\hline June & 517 \\
\hline July & 487 \\
\hline August & 511 \\
\hline September & 517 \\
\hline Total & 3,079 \\
\hline \multicolumn{2}{|c|}{ Source: Prepared by the author } \\
\hline
\end{tabular}

Out of the 14,321 production orders issued between April and September 2017, only 3,079 were analyzed, as shown in Table 3. By examining the data from such orders in the different Control systems, it was only possible to assess the Quality non-conformances recorded in the Systems Applications and Products (SAP) system and the handwritten formats; FlexTime does not count with the options to record defects.

The monthly Production orders were chosen randomly, by using the Excel function "=Rand ( )" taking into account the quantities mentioned in Table 3. In the Handwritten formats, there were 607 Quality nonconformances found, while in Systems Applications and Products (SAP) system only 194 defects were recorded. This information is shown in Figure 1, which presents the monthly distribution of the Quality non-conformances recorded in both Production control systems, compared to the quantity of released Production Orders.

Figure 1. Record of Quality non-conformances through the distinct Control systems over the quantity of released Production Orders per month

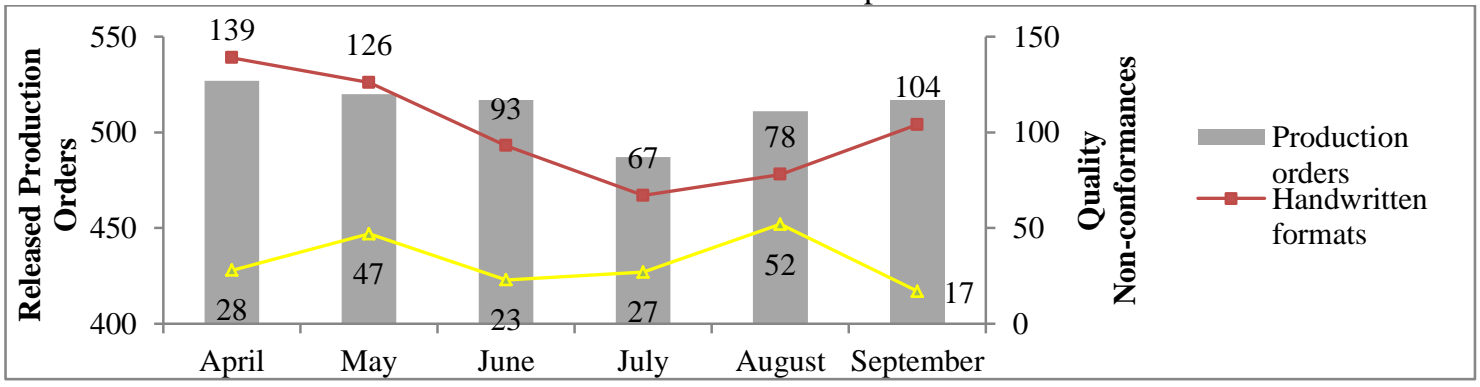

Source: Systems Applications and Products (SAP) system and handwritten formats 
In Figure 1 it is noticeable the fluctuation of the released Production orders and the oscillation on the recorded Non-conformances in both systems is different. Even though the defects found in the handwritten formats follow the released orders trend, it is evident that SAP recorded defects do not match the behavior of released Production orders.

Figure 2. Ratio of the quantity of defects recorded in handwritten formats and SAP records over the quantity of released Production orders per month

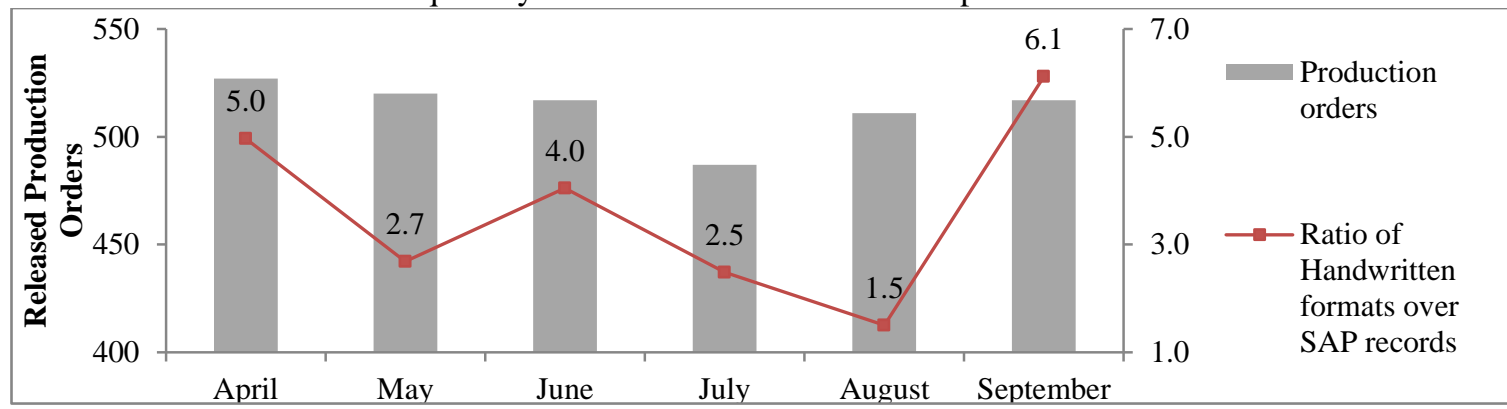

Source: Systems Applications and Products (SAP) system and handwritten formats

Figure 2 indicates that the tendency of the released Production orders is not related to the discrepancy between the Quality non-conformances records in both Control systems.

Figure 3: Record of Quality non-conformances in handwritten formats over released Production orders per month

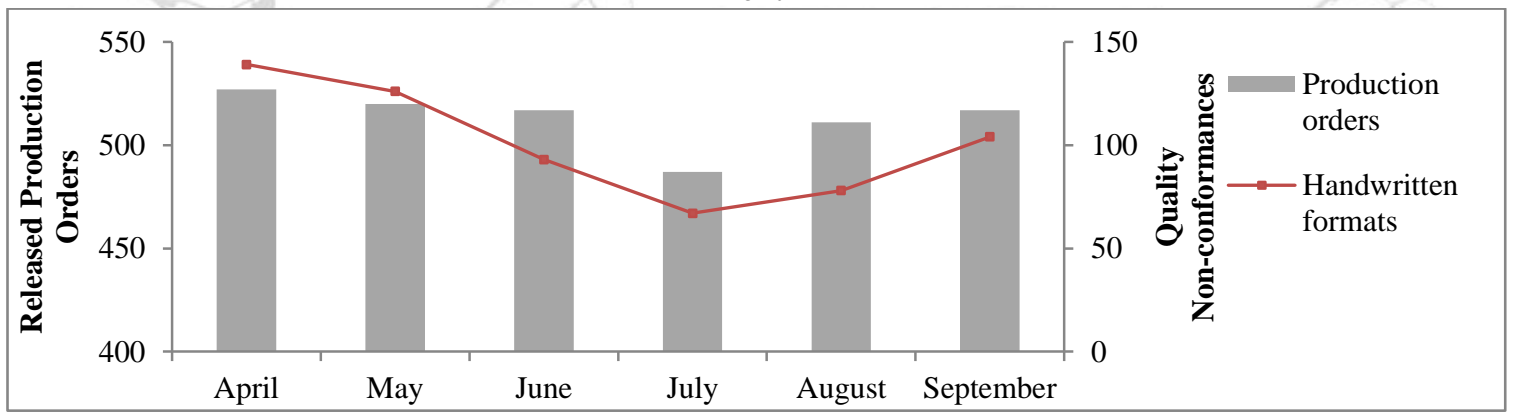

Source: Systems Applications and Products (SAP) system and handwritten formats

In order to get better notion about how the quantity of non-conformances fluctuates in both systems, two figures were generated. Figure 3 demonstrates once the production increases or diminishes the quantity of non-conformances increases and diminishes proportionally.

Figure 4: Record of Quality non-conformances in SAP over released Production orders per month

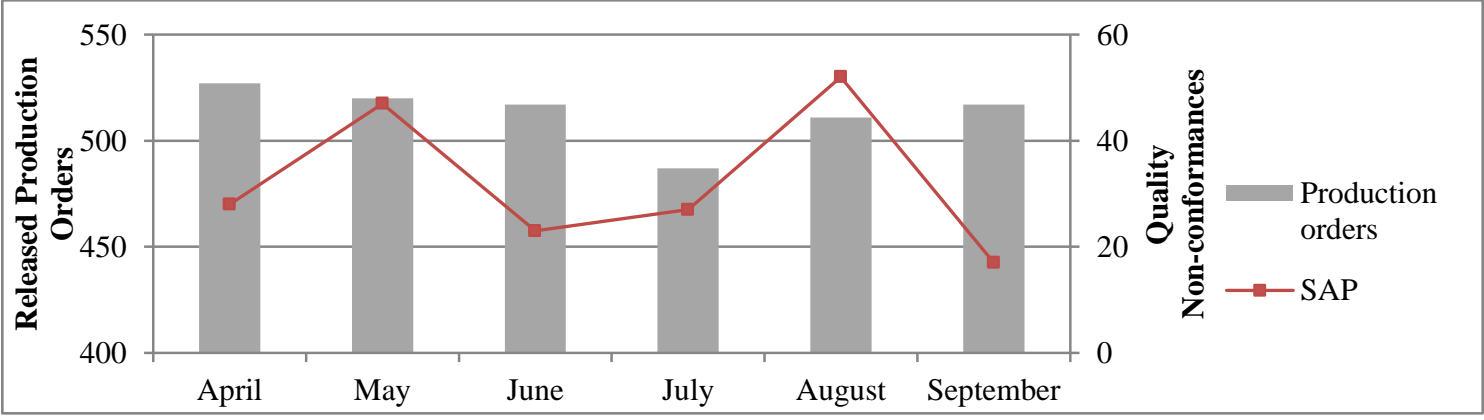

Source: Systems Applications and Products (SAP) system 
On the other hand, Figure 4 demonstrates the recorded non-conformances in the Systems Applications and Products (SAP) system does not show any correspondence with the quantity of released Production orders' monthly tendency.

\section{Discussion}

After analyzing the information obtained in the Results section, it is possible to say the objectives of the study were met as well as to define whether the hypothesis were accepted or rejected. By examining Figure 2 , it can be observed the ratio between handwritten and SAP records does not fluctuate with the change in the quantity of Production orders released to the Phosphoric Acid anodizing. In other words, the general hypothesis was rejected.

Contrariwise, Figure 1 proves there is a high discrepancy between the Quality non-conformances records kept in both Control systems, which means one of the systems is not capturing all required information from the production line. Based on the nature of the process and the lack of concordance between the records, it was decided that the Systems Applications and Products (SAP) records were not reliable. As observed in Figure 3, the quantity of Quality non-conformances found in the anodized components is directly proportional to the quantity of released Production orders; therefore the specific hypothesis 1 was accepted.

Due to the ease of recording and tracing of the Quality non-conformances it was expected that the electronic systems would contain more information. However, once the results were analyzed, it was proven the electronic Control systems do not have more information than the handwritten formats, concluding specific hypothesis 2 could be rejected.

\section{Conclusion}

After the gathered data was analyzed, it is strongly recommended that the subject Company defines and establishes the use of one single software that meets the Phosphoric Acid Anodizing area requirements. This way the information discrepancies between Production control systems would be eliminated and the traceability, reliability and quality of the data would consequently improve.

In case this recommendation was followed, the time used for filling handwritten forms out could be better used by the Operational and Quality departments' personnel. By improving the Control and the Quality of the data logging process in the Phosphoric Acid Anodizing area, production efficiency and effectiveness would be improved and information traceability would become completely reliable.

\section{References}

Cuatrecasas-Arbós, L., Fortuny-Santos, J., Ruiz-de-Arbulo-López, P., \& Vintró-Sanchez, C. (June 8, 2015). Monitoring processes through inventory and manufacturing lead time. Industrial Management \& Data Systems, pags. 951-970.

Di Natale, V. (2016). How to Fulfill AS 9100 Traceability Requirements as a Defense Subcontractor. Obtained from In Compliance: https://incompliancemag.com/

Eisler, M. N. (2017). Black Box: Personal Computing, the Notebook Battery Crisis, and Postindustrial Systems Thinking. Technology and Culture, 368-391.

García Pérez, A. (2006). Instalación de una Planta Moderna de Anodizado para Lima: Universidad Nacional Mayor de San Marcos.

Giudice, C. A., \& Pereyra, A. M. (2009). Proteccion de los materiales. Berisso.

Hufnagel, W. (1992). Manual del Aluminio Vol. II. España: Reverté.

Jiménez Luengo, E. I. (2010). Análisis de los sistemas de control de la producción Kanban y Conwip bajo escenarios. Sevilla: Escuela Técnica Superior de Ingenieros. 
Monette, F., \& Van Bogart, M. (2011). Trace, Track and Control: High Production Output at Low Costs. Obtained from Microscan Systems, Inc.: https://www.microscan.com/en-us.

Morris, W. (2011). The American Heritage Dictionary of the English Language. American Heritage Publishing Company.

Pennisi, M. S. (1999). Anodising. Obtained from Coatings \& Fabrication: https://www.coatfab.com/

Prado, J. R. (1992). Sistemas continuos o estandarizados. En La planeación y el control de la producción (pág. 17). Azcapotzalco: Universidad Autónoma Metropolitana.

Sippper, D., \& Bulfin, R. L. (1998). Planeación y control de la producción. D.F., México: McGraw-Hill.

Soto, D. (March 27, 2017). ¿Qué es SAP y para qué sirve SAP? Obtained from Nextech. 